\title{
Collision-Free Path Planning for Efficient Inspection of Free-Form Surface by Using a Trigger Probe
}

\author{
Zhengcai Zhao a, b, 1, Yao Li ${ }^{\mathrm{a}}$, Yucan Fu ${ }^{\mathrm{a}}$, Dawei Ding ${ }^{\mathrm{a}}$ \\ ${ }^{a}$ National Key Laboratory of Science and Technology on Helicopter Transmission, \\ Nanjing University of Aeronautics and Astronautics, Nanjing 210016, China \\ ${ }^{b}$ Jiangsu Key Laboratory of Precision and Micro-Manufacturing Technology, Nanjing \\ University of Aeronautics and Astronautics, Nanjing 210016, China
}

\begin{abstract}
Measurement path planning plays an essential role in on-machine measurement, which is often required to be time-saving and collision-free. This paper proposes a novel path planning methodology and develops an automatic collision avoidance strategy for measurement. Measurement points are generated by Contour section sampling(CSS), Grid mesh sampling(GMS), and Locally dense sampling (LDS) on the free-form surface. Afterwards, a shortest path algorithm (SPA) and a non-interference path algorithm (NIPA) are developed respectively. The simulations show that the proposed method can reduce the total inspection distance to nearly $7.82 \%$ than the original one. The presented path planning method can measure the surface of large aerospace parts directly by using a trigger probe.
\end{abstract}

Keywords. OMP, Path planning, Collision-free, Traveling-salesman problem.

\section{Introduction}

On-machine measurement refers to the integration of measuring equipment on the machine tool.[1] On-machine measurement with a touching probe (OMP) is mostly used in the machining process due to its high measuring accuracy and easy implementation.[2] The schematic of OMP system is presented in Fig.1, in which once the probe touches the part along the measuring direction, a signal is sent from the probe and is received by the wireless interface. The NC system is asked to record the positions of the grating rulers by the wireless interface, which can be used to compute the measurement data of the part.

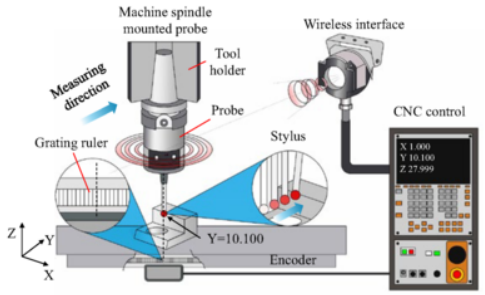

Fig. 1 Schematic of OMP system

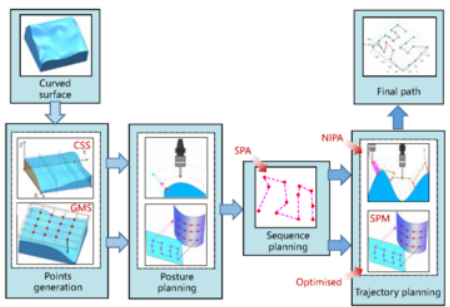

Fig. 2 Overview of the proposed path planning approach

\footnotetext{
${ }^{1}$ Corresponding Author. zhengcaizhao@nuaa.edu.cn
} 
Searching for the shortest inspection path is regularly formulated as a traveling salesman problem (TSP). TSP is to determine a minimum path which a traveling salesman can find so that he can visit all the cities without traveling through the same city more than once. Teodor et al.[3] treated the measurement path optimization for the car body parts inspection as a TSP. Zakharov et al.[4] modeled the optimal path of the touch sensor on CMM(coordinate measuring machine) based on the TSP solution. Stojadinovic et al. [5] optimized a measuring path of prismatic parts on a CMM based on TSP. Li et al.[6] used the TSP algorithm to generate the shortest inspection sequence for a 5-axis on-machine inspection (OMI) system. Cho and Seo[7] developed an inspection planning strategy for sculptured surfaces by using TSP algorithms.

Inspection collisions refer to the intersections between the machine tool, the part, and the probe which will damage to the OMP system. Zhao et al. [8] proposed a geometry-guide planning approach for generating a collision-free probing path. Lee and Park [9] presented a massively parallel and connectionist algorithm for collision-free path planning, which is based on a potential field and a collision penalty function generated by polyhedral obstacles. Li et al.[6] developed a convex hull-based algorithm to generate the collision-free measurement path.

The free-form surface is commonly seen in structural parts from the aerospace instead of conventional feature measurement (e.g., edges, planes, holes). Due to its immense size, it is necessary to inspect the structural parts with free-form surface quickly. Motivated by previous publications on integrating the minimization method and collision avoidance approach, we aim to generate a collision-free path for large free-form parts by using an on-machine trigger probe. The outline of this paper is organized as follows.

The overview of the proposed approach is presented in Section 2. Some results and related discussions are provided in Section 3. Finally, the conclusion of this paper is given in Section 4.

\section{Path planning principle for free-form surface}

This paper aims to offer an approach for planning a collision-free path for inspecting the freeform surface efficiently by using a trigger probe, which can decrease the measuring time as short as possible and guarantee the safety of the OMP system. The feed rate is set in a rational range to meet the requirements of the limitation of the machine tool and dynamic issues, which will not be discussed in this paper.

The overview of the proposed method is presented in Fig.2. The contour section sampling (CSS), grid mesh sampling (GMS), and Locally dense sampling (LDS) are introduced to generate the distributed measurement points based on the nominal shape of the part for the different applications at the beginning. Afterwards, the postures of the trigger probe for each measurement point from CSC, GMS, and LDS are designed, and their related points are computed to locate the probe are computed. The shortest path algorithm (SPA) is developed to optimize the measuring path for decreasing the length of detecting paths. A non-interference path algorithm (NIPA) is developed to avoid the collision of the original measurement path, and an optimized safe-plane based method(SPM)[24] is used to prevent the crash of the measurement path from SPM. Finally, the shortest collision-free path for free-form surface inspection is output. 


\subsection{Points Distribution and Measurement Posture}

a) Points Distribution

CSS is firstly used to compute a series of intersecting curves between the free-form surface $\mathrm{s}$ and several section planes along a specific direction; afterwards, the points are sampled from these intersecting curves (cp. Fig3(a)). In this way, the samplings of threedimensional (3D) free-form surface are transferred into a group of the samplings of 2D curves, which are easily implemented. [10] GMS is used to build a plane mesh with the environment of the designed shape of the part, and project the grid points onto the designed shape $[11,12]$. Each point of the intersection between the mesh grid and the designed shape of part would specify a measurement point (cp. Fig.3(b)). It can be seen from Fig.3(c) that it is not easy for both CSS and GMS to increase the density of the measurement points in the local region of the part.

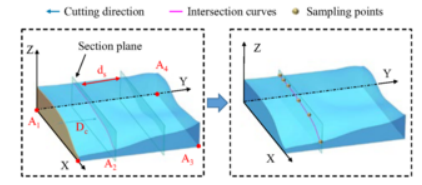

a)

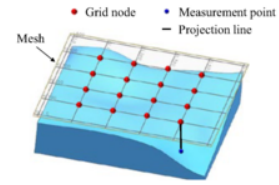

b)

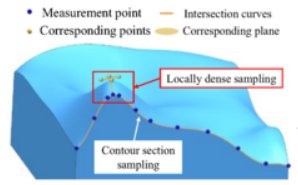

c)

Fig. 3 Points Distribution for the free-form surface a) CSS b)GMS c)LSD

b) Posture definition

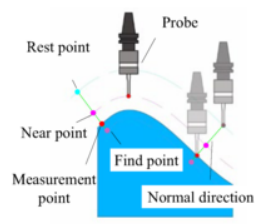

a)

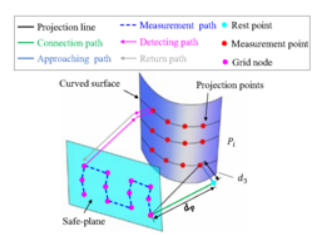

b)

Fig. 4 Posture planning for measurement points a) CSS Posture b)GMS Posture

Since the measurement points have been generated based on the nominal shape of the part, it is necessary to plan the posture for each measurement point. Posture planning is used to determine the approaching path and direction for each measurement point.

\subsection{Sequence and Trajectory planning}

The inspection path of OMP consists of the approaching and traveling path of the measurement points. The length of the OMP inspection path is majorly decided by the length of the traveling path. It is known that the sequence of the measurement points decides its traveling length. Hence, the optimization of the measurement point sequence is always carried out to shorten the traveling length, which can be regarded as a traveling salesman problem.

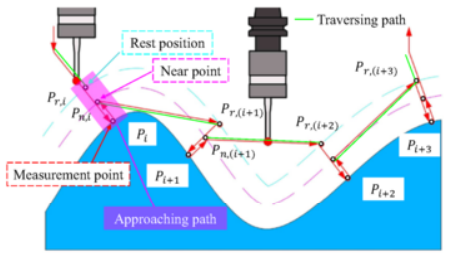

a)

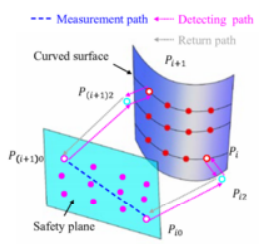

b)

Fig. 5 Sequence planning a)CSS b)GMS 
By solving TSP, an optimized sequence of the measurement points can be obtained. Ant colony optimization (ACO) algorithm is used to solve the optimization problems. The ants are found to follow the same path by tracing the pheromone laid by them. The sequence planning was simulated as ants foraging. In the ant system, an ant $k$ at node $i$ selects the next possible node $j$ with probability computed through the Z-axis random proportional rule. It can be defined as

$$
p_{i j}^{k}=\left\{\begin{array}{cl}
\frac{\tau_{i j}{ }^{\alpha} \eta_{i j}{ }^{\beta}}{\sum_{j \in \Lambda} \tau_{i j}{ }^{\alpha} \eta_{i j}{ }^{\beta}} & \text {, if } \mathrm{j} \in \Lambda \\
0 & , \text { other }
\end{array}\right.
$$

where $\tau_{i j}$ is the amount of pheromone on edge $(i, j), \alpha$ and $\beta$ are parameters that determine the relative influence of the pheromone trail and the heuristic information. $N_{i}^{k}$ is the feasible neighborhood of ant $k$ at point $i$, and $\Lambda$ is the set of points that ant $k$ has not visited yet. According to the recommendations for ant system technique, the following values are adopted: $\alpha=1, \beta=5$. In the ACO application, to obtain the optimal path, the ant colony with $m=500$ ants was used in 500 iterations.

\section{Simulation of a measuring probe path}

We just use one typical part that used in inspection zone to verify the proposed SPA and NIPA method. The testing part is shown in Fig.6a, whose surface fluctuates a lot. Twenty points were sampled from Part 1 by using the CSS method(cp. Fig. 6(b)).

The Zigzag method connects the neighbouring measurement points in order (cp. Fig. 6(c)), which is simple in concept. However, the collisions were detected when the probe moves from the two adjacent measurement points in a straight path(refer to Fig. 6(d)). There is still one crash that happens in the detecting path of testing part after the SPA method. The NIPA steps for collision detection and collision-avoidance is shown in Fig. 7 as collision detection, path projection, profile offsetting, automatic check. Finally it turns out a collision-free path. The results are shown in Table 1.

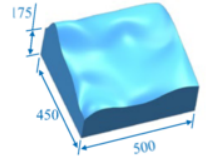

a)

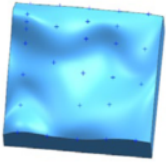

b)

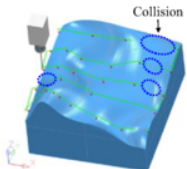

c)

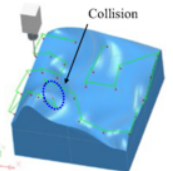

d)

Fig. 6 a)Testing part b) Distribution of measurement points c)Zigzag results d)SPA

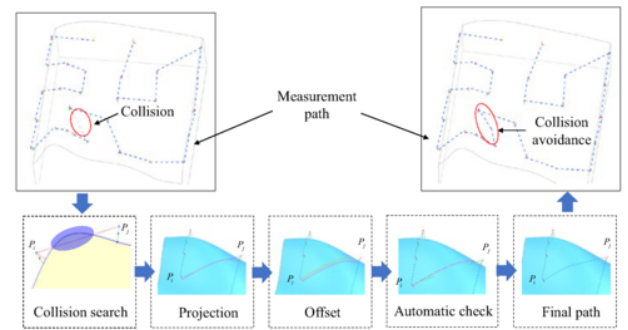

Fig. 7 Schematic of collision avoidance with NIPA on testing part 
The lengths of planned measurement paths by using the Zigzag and the proposed path(combining SPA and NIPA) are $3560.4 \mathrm{~mm}$ and $3282 \mathrm{~mm}$ for testing part. The detailed comparison results are given in Tab.1, which shows that the path lengths decrease $7.82 \%$ by using the proposed pathwhen compared with the Zigzag method.

Table 1. Comparison results of measurement path by using Zigzag and the proposed method

\begin{tabular}{ll}
\hline Results & Test part \\
\hline $\mathrm{D}_{\text {zigzag }}(\mathrm{mm})$ & 3560.4 \\
Collision number & 4 \\
$\mathrm{D}_{\text {SPA }}(\mathrm{mm})$ & 3278.2 \\
Collision number & 1 \\
$\mathrm{D}_{\text {total }}(\mathrm{mm})$ & 3282 \\
$\mathrm{D}_{\text {total }} / \mathrm{D}_{\text {zig }}$ & $92.18 \%$ \\
$\mathrm{I}_{1}=1-\mathrm{D}_{\text {total }} / \mathrm{D}_{\text {zigzag }}$ & $7.82 \%$ \\
\hline
\end{tabular}

\section{Conclusions}

This article presents a practical path planning method to plan the measurement path of OMP for large-scale free-form parts and improves the efficiency of on-machine measurement. The following conclusions can be drawn.

1) Contour section sampling, Grid mesh sampling, and Locally dense sampling are discussed for the generation of measurement points. LDS intends to increase the points number with a circle sequence selectively for parts registration.

2) The sequence planning establishes the measurement path for the free-form surface with the shortest path algorithm(SPA), which minimizes the total inspection path. In the trajectory planning stage, a non-interference path algorithm(NIPA) is carried out to determine each planned path with no collision.

3) The SPA and NIPA contribute to our proposed method, which can reduce the total inspection path to $7.82 \%$ comparing to the Zigzag path.

\section{Acknowledgements}

This work is partially supported by National Natural Science Foundation of China (No.51805258), Natural Science Foundation of Jiangsu Province (No. BK20180441).

\section{Appendices}

No

\section{References:}

[1]. Nishida, I., M. Murase and K. Shirase, Sequence planning of on-machine measurement and re-machining. Journal of Advanced Mechanical Design, Systems, and Manufacturing, 2019. 13(1): p. JAMDSM0014JAMDSM0014.

[2]. Sepahi-Boroujeni, S., J. Mayer and F. Khameneifar, Repeatability of on-machine probing by a five-axis machine tool. International Journal of Machine Tools and Manufacture, 2020: p. 103544. 
[3]. Teodor, V.G., et al., Optimization of the measurement path for the car body parts inspection. Measurement, 2019. 146: p. 15-23.

[4]. Zakharov, O.V., A.F. Balaev and A.V. Kochetkov, Modeling Optimal Path of Touch Sensor of Coordinate Measuring Machine Based on Traveling Salesman Problem Solution. Procedia Engineering, 2017. 206: p. 1458-1463.

[5]. Stojadinovic, S.M., et al., Ants Colony Optimisation of a Measuring Path of Prismatic Parts on a CMM. Metrology and Measurement Systems, 2016. 23(1): p. 119-132.

[6]. Li, Y., et al., Orientation-point relation based inspection path planning method for 5-axis OMI system. Robotics and Computer-Integrated Manufacturing, 2020. 61: p. 101827.

[7]. Cho, M.W. and T.I. Seo, Inspection Planning Strategy for the On-Machine Measurement Process Based on CAD/CAM/CAI Integration. International Journal of Advanced Manufacturing Technology, 2002. 19(8): p. 607-617.

[8]. Zhao, H., et al., Automated dimensional inspection planning using the combination of laser scanner and tactile probe. Measurement, 2012. 45(5): p. 1057-1066.

[9]. Lee, S. and J. Park, Neural computation for collision-free path planning. Journal of Intelligent Manufacturing, 1991. 2(5): p. 315-326.

[10]. He, G., et al., CAD-based measurement planning strategy of complex surface for five axes on machine verification. The International Journal of Advanced Manufacturing Technology, 2017. 91(5-8): p. 21012111.

[11]. Li, Y. and P. Gu, Free-form surface inspection techniques state of the art review. Computer-Aided Design, 2004. 36(13): p. 1395-1417.

[12]. Ainsworth, I., M. Ristic and D. Brujic, CAD-Based Measurement Path Planning for Free-Form Shapes Using Contact Probes. International Journal of Advanced Manufacturing Technology, 2000. 16(1): p. 23-31. 